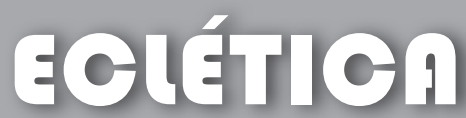 química
}

\section{ANÁLISE CONFORMACIONAL DE COMPOSTOS DE BIGINELLI COM ATIVIDADE ANTINEOPLÁSICA}

\author{
M. V. Marques, D. Russowsky, L. A. M. Fontoura \\ Curso de Química, Centro de Educação Ciências e Artes, Universidade Luterana do Brasil (ULBRA). \\ Av. Farroupilha, 8001. CEP 92425-900 Canoas - RS \\ Departamento de Química Orgânica, Instituto de Química, Universidade Federal do Rio Grande do Sul (UFRGS). \\ Cp15003, CEP 91501-970 Porto Alegre - RS \\ marcelovolp@hotmail.com
}

\begin{abstract}
Resumo: Diidropirimidinonas são heterociclos com atividade antineoplásica conhecida. O monastrol e alguns análogos são exemplos. A análise conformacional representa uma etapa preliminar importante em estudos que visam correlacionar a estrutura do composto com sua atividade. Neste trabalho, descrevemos a análise conformacional do monastrol e diversos análogos por cálculo semi-empírico AM1 e ab initio HF/6-31G*. Quatro geometrias de equilíbrio foram encontradas ( $s$-cis/ap, s-cis/sp, s-trans/ap e s-trans/sp), tendo como rotações internas importantes a do sistema carbonilado $\alpha, \beta$-insaturado e a do grupo arila ligado ao heterociclo.
\end{abstract}

Palavras-chave: análise conformacional, diidropirimidinonas, compostos de Biginelli, AM1, HF/6-31G*

\section{Introdução}

Em 1893, o químico italiano Pietro Biginelli descobriu uma reação multicomponente que produzia diidropirimidinonas multifuncionalizadas (1), DHPM, em um simples processo de uma única operação (esquema 1) [1].<smiles>O=C[Al]</smiles><smiles>[X]C1=C(C)C([R])=C(CC)NC1</smiles>

$\mathrm{R}=$ alquila, $\mathrm{H}$

$\mathrm{Ar}=$ arila

$E=$ grupo retirador de elétrons $\mathrm{X}=\mathrm{O}, \mathrm{S}$

Esquema 1. Síntese de diidropirimidinonas multifuncionalizadas (1) de Biginelli.
Desde os anos 80, o interesse em DHPMs foi aumentado significativamente, devido, em particular, a sua similaridade estrutural com as diidropiridinas (2), DHP, conhecidas como moduladores do canal de cálcio (figura 1) [2].<smiles>[X]C1=CNC([Al])C(F)=C1[R]</smiles><smiles>[R]C1=C(F)C(Br)C(F)=C([R])N1</smiles><smiles>COC1CNC(=O)NC1c1ccccc1Cl</smiles>

1 DHPM 2 DHP

3 Conformação bioativa (DHPM)

$$
\begin{aligned}
& \mathrm{R}, \mathrm{R}^{\prime}=\text { alquila, } \mathrm{H} \\
& \mathrm{Ar}=\text { arila } \\
& \mathrm{E}=\text { grupo retirador de elétrons } \\
& \mathrm{X}=\mathrm{O}, \mathrm{S}
\end{aligned}
$$

Figura 1. Estruturas de diidropirimidinonas (1), diidropiridinas (2) multifuncionalizadas e da conformação bioativa da DHPM (3). 
Foi observado que DHPMs exibiam similaridade farmacológica com a DHP. Mais recentemente, foi constatado que DHPMs não serviam somente como moduladores do canal de cálcio, mas também possuíam atividade biológica como inibidores do câncer de próstata [2].

O monastrol (4a) é um exemplo de diidropirimidinona, cuja capacidade de afetar a divisão celular é bastante conhecida, sendo assim um potencial agente anticâncer [3,4]. Russowsky e colaboradores [4] descreveram a síntese do monastrol (4a) e de onze análogos e determinaram a atividade antiproliferativa em sete diferentes linhagens de células humanas. Entre as células testadas estão as de mama, melanoma, rim, ovário e próstata. Os compostos são apresentados na figura 2 .
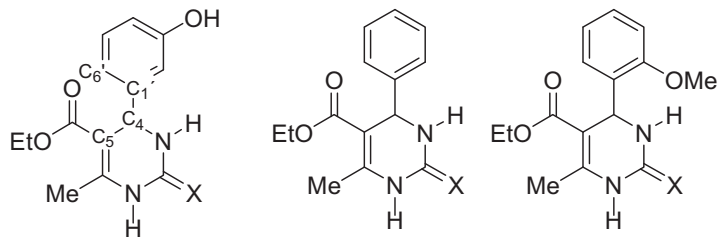

$$
\begin{aligned}
& \begin{array}{lll}
\mathbf{4 a}-X=S \text { (monastrol) } & 5 \mathbf{a}-X=S & 6 \mathbf{a}-X=S \\
\mathbf{4 b - X}=\mathrm{O} \text { (oxo-monastrol) } & 5 \mathbf{b}-X=O & \mathbf{6 b}-X=O
\end{array} \\
& \begin{array}{l}
7 a-X=S \\
7 b-X=O
\end{array} \\
& \begin{array}{l}
8 a-X=S \\
8 b-X=O
\end{array} \\
& 9 a-X=S \\
& 9 \mathrm{~b}-X=O
\end{aligned}
$$

Figura 2. Estruturas do monastrol (4a) e de seus análogos com atividade antineoplásica.

A análise conformacional consiste na interpretação ou predição das propriedades físicas ou químicas de uma determinada molécula através das suas variadas conformações [5]. Entre outras informações, a análise conformacional permite o conhecimento e descrições detalhadas das geometrias de equilíbrio. Estas informações são importantes em Química Medicinal, pois estes confôrmeros indicam de que forma os grupamentos funcionais estão orientados, descobrindo assim aspectos relevantes de como a molécula pode interagir com um receptor específico, uma vez que a conformação mais estável deve estar em maior número durante o processo de interação com o receptor. Entretanto, existe uma restrição quanto a estas informações porque não há necessariamente relação entre a conformação mais estável e a conformação bioativa, pois a geometria de equilíbrio pode sofrer mudanças na sua conformação de origem no momento da sua aproximação com o sítio receptor [6].

Uma proposta de conformação bioativa para diidropirimidinonas (3) que atuam como moduladores do canal de cálcio surgiu em 1995 (Figura 1). A configuração absoluta do carbono quiral é importante porque a atividade agonista/antagonista é dependente da orientação do grupo arila que atua como uma chave molecular entre elas. $\mathrm{O}$ heterociclo assume uma conformação próxima a bote orientando o grupo arila na posição axial, o qual bissecciona o ângulo formado entre o $\mathrm{C} 4$ e seus vizinhos no anel. $\mathrm{O}$ substituinte do grupo arila posiciona-se synperiplanar $(s p)$ em relação ao hidrogênio ligado a $\mathrm{C} 4$. O sistema carbonílico $\alpha, \beta$-insaturado do grupo éster assume orientação $s$-cis [7].

Em 1997, Kappe e colaboradores [7] descreveram o primeiro estudo conformacional destes compostos obtido por modelagem molecular. Os métodos utilizados foram o ab initio $\mathrm{HF} / 3-21 \mathrm{G}^{*} \mathrm{e}$ os semi-empíricos AM1 e PM3. Três dos compostos estudados (10-12) são apresentados no esquema 2.

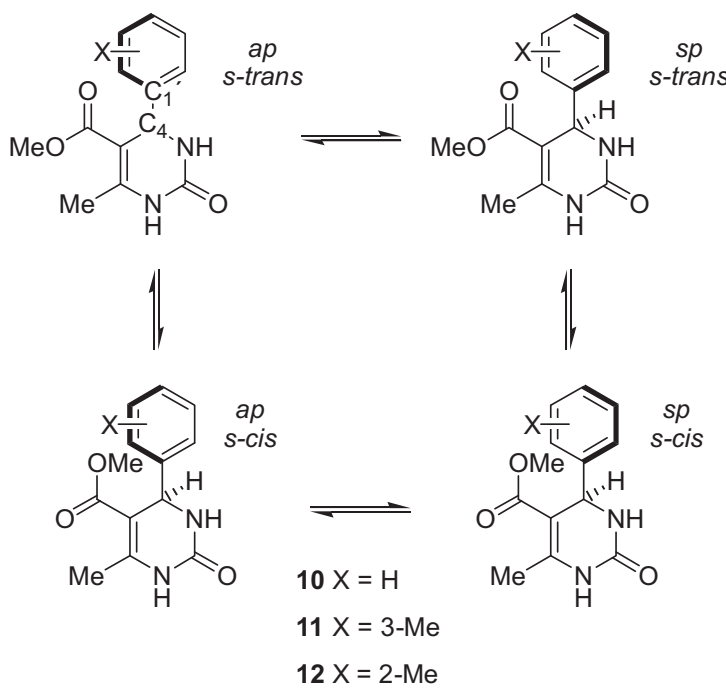

Esquema 2. Geometrias de equilíbrio de uma 4-aril5-metoxicarbonildiidropirimidinona. 
As geometrias de equilíbrio propostas resultam da rotação do grupo arila e do sistema carbonílico $\alpha, \beta$-insaturado. Com relação ao substituinte do primeiro grupo, são duas as geometrias de equilíbrio, antiperiplanar (ap) e synperiplanar $(s p)$, relacionadas com o hidrogênio do $\mathrm{C} 4$. O sistema carbonílico $\alpha, \beta$-insaturado por sua vez apresenta duas conformações de mínima energia, $s$-trans e $s$ cis. Um exemplo é mostrado no esquema 2 [7]. As conformações de menor energia foram encontradas com o grupo éster orientado $s$-cis e o substituinte do grupo arila $s p$. Diferenças significativas de energia entre as quatro geometrias de equilíbrio, entretanto, apenas são observadas no caso do grupo arila ortosubstituído (12). Segundo os autores, a conformação bioativa corresponde ao confôrmero de menor energia $s$-cis/sp [7].

As análises conformacionais completas das rotações do sistema carbonílico $\alpha, \beta$-insaturado e do grupo arila foram realizadas pelos hamiltonianos AM1 e PM3. Segundo o cálculo AM1, na rotação da fenila são encontrados dois mínimos de energia com ângulos de diedro C5-C4-C1'-C6' iguais a 75' (ap) e $240^{\circ}(s p)$, sendo este último $2,3 \mathrm{kcal} \mathrm{mol}^{-1}$ mais estável que o primeiro. A barreira energética na conversão $s p \longrightarrow a p$ foi estimada em $5,9 \mathrm{kcal} \mathrm{mol}^{-1}$. Para a rotação do grupo éster, a barreira energética para passar de s-cis para s-trans, as duas geometrias de equilíbrio, foi de $3,9 \mathrm{kcal} \mathrm{mol}^{-1}$, sendo o primeiro deles $0,4 \mathrm{kcal} \mathrm{mol}^{-1}$ mais estável, apresentando ângulos de diedro apenas um pouco fora do plano, de $15^{\circ}$ e $210^{\circ}$ respectivamente [7].

Neste trabalho foram realizadas as análises conformacionais do monastrol (4a) e de seus análogos (5a-9a e 4b-9b). A otimização de geometria para cada conformação dos compostos estudados foi realizada com o hamiltoniano AM1. As quatro geometrias de equilíbrio de cada composto foram novamente otimizadas por cálculo HF no conjunto de funções de base $6-31 \mathrm{G}^{*}$. A energia total de cada confôrmero foi utilizada para cálculo de sua fração molar no equilíbrio conformacional.

\section{Parte Computacional}

Os cálculos foram executados nos seguintes computadores: Toshiba com processador Celeron ${ }^{\circledR}$ em $1.80 \mathrm{GHz}$ com memória RAM de $256 \mathrm{MB}$ e disco rígido de $30 \mathrm{~GB}$ e um PC com Processador Intel ${ }^{\circledR}$ Pentiun ${ }^{\circledR} 4$ CPU $2.60 \mathrm{GHz}, 512 \mathrm{MB}$ de RAM disco rígido $80 \mathrm{~GB}$. O sistema operacional usado em ambos foi o Microsoft Windows XP Professional Versão 2002. Foram realizados cálculos pelo hamiltoniano AM1 [8] e ab initio Hartree-Fock no conjunto de bases 6-31G* contidos no programa Spartan '02 (Versão 1.0.1) [9].

Otimizações de geometria foram realizadas para as conformações de cada composto estudado (4a-9a e 4b-9b) pelo método semi-empírico AM1 para ângulos de diedro $\theta$ C5-C4-C1'-C6' com restrições de 0 a $360^{\circ}$ com incrementos de $20^{\circ}$ (figura 2 ). A análise conformacional foi realizada para as duas geometrias do sistema carbonílico $\alpha, \beta$ insaturado, $s$-cis e s-trans. A mesma análise conformacional foi feita para os compostos $4 \mathbf{a}$ e $4 \mathbf{b}$ usando o cálculo ab initio HF no conjunto de funções de base 6-31G*. Na etapa seguinte, as quatro geometrias de equilíbrio de cada composto foram completamente otimizadas, agora livres de qualquer restrição de ângulo torcional pelo método HF/6-31G*.

\section{Resultados e Discussão}

A liberdade conformacional dos compostos de Biginelli resulta, principalmente, da rotação de três ligações, a saber, a ligação do éster, do sistema carbonílico $\alpha, \beta$-insaturado e da arila com o heterociclo. Ácidos carboxílicos e seus derivados apresentam duas geometrias de equilíbrio $Z$ e $E$ devido à ressonância da carbonila com o heteroátomo vizinho. No caso de ésteres, o equilíbrio desloca-se totalmente no sentido da primeira delas, na qual o grupo $\mathrm{R}$ da alcoxila será estabilizado em função da carga parcial do oxigênio ser negativa e a sua positiva [5]. Assim como o grupo éster, o sistema carbonílico a,binsaturado também faz ressonância, o que determina a sua planaridade e origina duas conformações mais estáveis, $s$-cis e $s$-trans [5]. O problema resume-se, portanto, em avaliar a rotação do grupo arila para cada uma das geometrias de equilíbrio do sistema carbonílico $\alpha, \beta$-insaturado, mantendo o éster na conformação Z.

Na primeira parte da análise conformacional, o hamiltoniano AM1 foi utilizado em razão do seu baixo custo computacional, embora não seja o melhor método de cálculo para esta tarefa. A dificuldade deste método na determinação de barreiras rotacionais ocorre pela falta de dados experimentais para estados de transição, uma vez que os parâmetros que alimentam esse conjunto de informações são obtidos a partir das geometrias de equilíbrio [8]. 
Curvas relacionando o calor de formação com o ângulo torcional para o diedro C5-C4-C1'-C6' foram obtidos. Um exemplo típico é mostrado na figura 3 para o composto 4a. O gráfico apresenta duas curvas que correspondem à rotação do grupo arila nas conformações $s$-cis e $s$-trans do éster conjugado. A análise conformacional indica dois mínimos e dois máximos de energia para a rotação do grupo arila. As geometrias de equilíbrio são aquelas com ângulos torcionais de $100^{\circ}$ e $280^{\circ}$, as quais apresentam pequena diferença de energia. A conversão $a p \longrightarrow s p$ pode ocorrer via dois diferentes estados de transição com diedros de $40^{\circ}$ e $220^{\circ}$. As barreiras rotacionais foram estimadas em aproximadamente $2,5 \mathrm{kcal} \mathrm{mol}^{-1}$. Qualquer que seja a inclinação da fenila, o rotâmero $s$ cis é sempre mais estável que o $s$-trans.

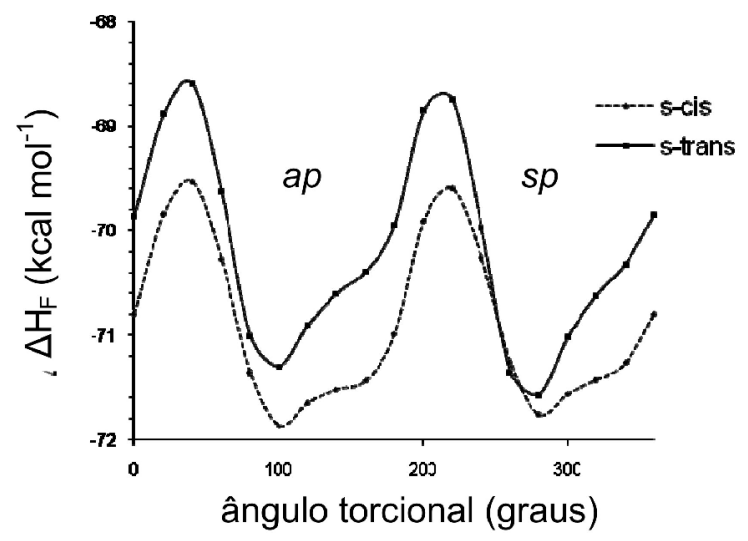

Figura 3. Calor de formação $\left(\mathrm{kcal} \mathrm{mol}^{-1}\right) \mathrm{x}$ ângulo torcional (graus) para a rotação do grupo arila (C5C4-C1'-C6') nas conformações s-cis e s-trans da carbonila $\alpha, \beta$-insaturada do monastrol (4a).AM1

O gráfico apresentado na figura 4 mostra as energias relativas dos rotâmeros dos compostos 5a, com a arila não substituída, e 6a, 4a e 7a, com substituinte em orto, meta e para respectivamente. Diferente do que acontece com os demais, no composto 6a, o rotâmero $s p$ apresenta ângulo de diedro de $340^{\circ}$. Uma maior diferença energética ocorre entre as geometrias de equilíbrio, sendo o rotâmero $s p 2,2 \mathrm{kcal} \mathrm{mol}^{-1}$ mais estável. As conformações de máxima energia são aquelas com ângulos torcionais de $60^{\circ} \mathrm{e} 220^{\circ}$. As barreiras rotacionais na conversão de $a p \longrightarrow s p$ foram estimadas em 2,6 e 6,3 $\mathrm{kcal} \mathrm{mol}^{-1}$. Resultados semelhantes obtidos no mesmo hamiltoniano foram observados por Kappe [7] para compostos análogos, tendo como substituinte na arila uma metila em posição orto ou meta (10-12).

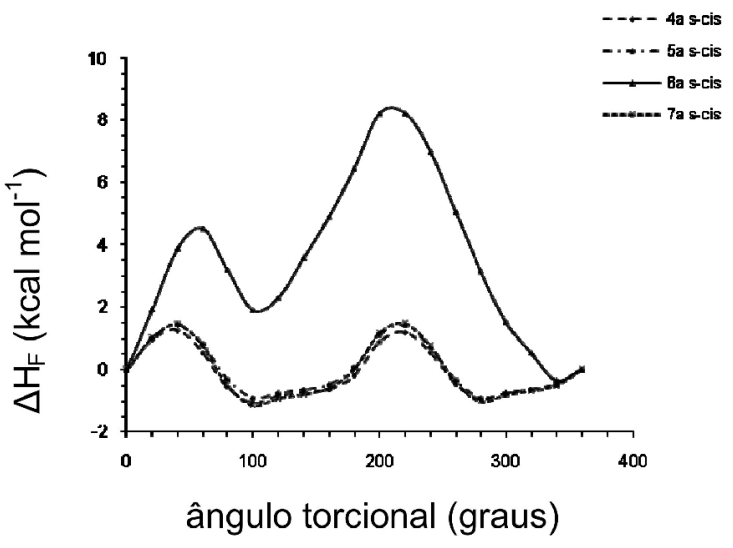

Figura 4. Calor de formação (kcal mol $\left.{ }^{-1}\right) \mathrm{x}$ ângulo torcional (graus) para a rotação do grupo arila (C5C4-C1'-C6') dos compostos 4a-7a, na conformação $s$-cis da carbonila $\alpha, \beta$-insaturada. AM1

Nas geometrias de equilíbrio, a fenila bissecciona o ângulo C5-C4-N3. Apesar de a fenila estar eclipsada com o hidrogênio de $\mathrm{C} 4$, a distância em relação aos demais ligantes deste último será maior, semelhante ao observado no isoproprilbenzeno [5]. O máximo de energia será dado quando, ao contrário do caso anterior, a fenila estiver mais próxima de $\mathrm{C} 5$ e N3.

Como já foi discutido, o composto 6a, apresenta uma geometria de equilíbrio $s p$ com diedro de $340^{\circ}$, diferente do que ocorre com os demais, nos quais o rotâmero $s p$ mais estável é o de $280^{\circ}$. Isto se deve ao fato do grupo substituinte na posição orto forçar a carbonila a sair da planaridade com o heterociclo, perdendo assim a ressonância e desestabilizando o confôrmero. Portanto, ao invés da arila bisseccionar o heterociclo, ocorre uma torção de diedro de mais $60^{\circ}$.

Outro detalhe que a análise conformacional para o composto orto substituído 6a mostra é a diferença de energia entre seus dois estados de transição, com diedros $60^{\circ}$ e $220^{\circ}$. Este último é 3,7 $\mathrm{kcal} \mathrm{mol}^{-1}$ mais energético que o primeiro, uma vez que o grupo metóxi força a carbonila para fora do plano do heterociclo da mesma forma como ocorre com o confôrmero de $280^{\circ}$. Resultados semelhantes foram obtidos para a série do oxo-monastrol $(\mathrm{X}=\mathrm{O})$.

A análise conformacional do monastrol (4a) e do oxo-monastrol (4b) foi também realizada através de cálculo ab initio Hartree-Fock no conjunto de funções de base $6-31 \mathrm{G}^{*}$. A figura 5 exibe as duas curvas de análise conformacional para o monastrol 


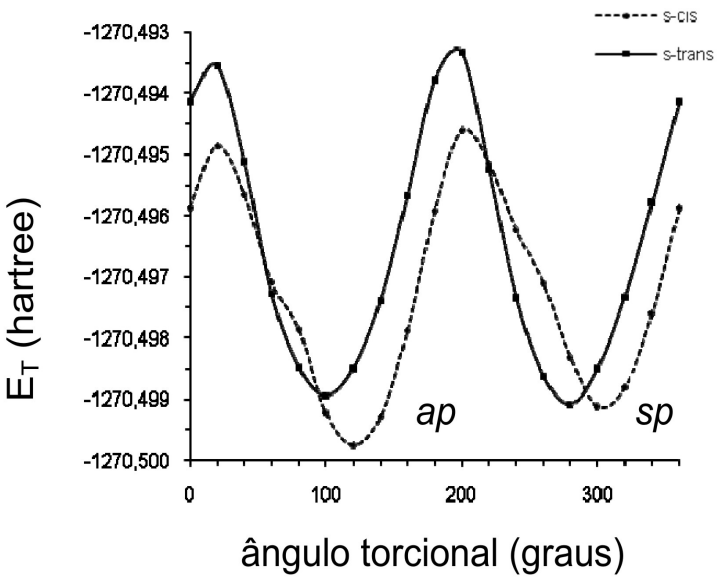

Figura 5. Análise conformacional das geometrias $s$ cis e s-trans do monastrol (4a) por cálculo HF/6$31 \mathrm{G}^{*}$. (4a). Como esperado, dois mínimos e dois máximos de energia foram encontrados em cada curva. As conformações de máxima energia apresentam ângulos de diedro $20^{\circ}$ inferiores àquelas obtidas pelo método AM1. As conformações de equilíbrio para a geometria $s$-trans se assemelham às encontradas pelo cálculo semi-empírico. Na geometria $s$-cis, por sua vez, diedros $20^{\circ}$ maiores foram encontrados. As barreiras rotacionais foram encontradas próximas a 3 $\mathrm{kcal} \mathrm{mol}^{-1}$, pouco superiores às obtidas pelo método AM1. Apenas para comparação, a barreira encontrada na ligação C2-C3 no butano $[10,11]$ é de $5,5 \mathrm{kcal} \mathrm{mol}^{-1}$.

A análise conformacional realizada pelo cálculo ab initio indica as mesmas quatro conformações de equilíbrio obtidas pelo semi-empírico. Estes quatro rotâmeros foram tomados como verdadeiros para todos os compostos das duas séries. Os ângulos

Tabela 1. Ângulo de diedro e fração molar $\left(\mathrm{x}_{\mathrm{i}}\right)$ para cada uma das quatro geometrias de equilíbrio dos compostos 4a-9a e 4b-9b (HF/6-31G*).

\begin{tabular}{|c|c|c|c|c|c|}
\hline \multicolumn{3}{|c|}{ Série do monastrol (4a) } & \multicolumn{3}{|c|}{ Série do oxo-monastrol (4b) } \\
\hline Composto & Diedro (graus) & $\mathrm{x}_{\mathrm{i}}(300 \mathrm{~K})$ & Composto & Diedro (graus) & $\mathrm{x}_{\mathrm{i}}(300 \mathrm{~K})$ \\
\hline 4a $s$-cis (ap) & 122 & 0,43 & $\mathbf{4 b} s$-cis (ap) & 126 & 0,45 \\
\hline $4 \mathbf{a} s$-cis $(s p)$ & 305 & 0,21 & 4b $s-c i s(s p)$ & 307 & 0,26 \\
\hline 4a $s$-trans (ap) & 102 & 0,17 & 4b $s$-trans (ap) & 105 & 0,11 \\
\hline $\mathbf{4 a} s$-trans $(s p)$ & 280 & 0,19 & $\mathbf{4 b} s$-trans $(s p)$ & 279 & 0,18 \\
\hline $5 \mathbf{a} s-c i s(a p)$ & 124 & 0,37 & $\mathbf{5 b} s-c i s(a p)$ & 125 & 0,40 \\
\hline $5 \mathbf{a} s-c i s(s p)$ & 302 & 0,37 & $\mathbf{5 b} s-c i s(s p)$ & 303 & 0,40 \\
\hline $\mathbf{5 a} s$-trans $(a p)$ & 108 & 0,13 & $\mathbf{5 b} s$-trans $($ ap $)$ & 109 & 0,10 \\
\hline 5a $s$-trans $(s p)$ & 288 & 0,13 & $\mathbf{5 b} s$-trans $(s p)$ & 288 & 0,10 \\
\hline 6a $s$-cis (ap) & 116 & 0,12 & $\mathbf{6 b} s$-cis (ap) & 115 & 0,38 \\
\hline $\mathbf{6 a} s$-cis $(s p)$ & 349 & 0,75 & $\mathbf{6 b} s-c i s(s p)$ & 348 & 0,26 \\
\hline 6a $s$-trans (ap) & 113 & 0,04 & $\mathbf{6 b} s$-trans (ap) & 112 & 0,11 \\
\hline 6a $s$-trans $(s p)$ & 348 & 0,09 & $\mathbf{6 b} s$-trans $(s p)$ & 348 & 0,26 \\
\hline 7a $s$-cis (ap) & 126 & 0,43 & $7 \mathbf{b} s$-cis (ap) & 128 & 0,42 \\
\hline $7 \mathbf{a} s-c i s(s p)$ & 304 & 0,43 & 7b $s-c i s(s p)$ & 305 & 0,47 \\
\hline 7a s-trans (ap) & 107 & 0,06 & $7 \mathbf{b} s$-trans (ap) & 108 & 0,04 \\
\hline 7a $s$-trans $(s p)$ & 290 & 0,08 & $7 \mathbf{b} s$-trans $(s p)$ & 291 & 0,07 \\
\hline 8a $s$-cis $(a p)$ & 122 & 0,39 & $\mathbf{8 b} s$-cis $(a p)$ & 125 & 0,38 \\
\hline $\mathbf{8 a} s-c i s(s p)$ & 304 & 0,09 & $\mathbf{8 b} s-c i s(s p)$ & 307 & 0,13 \\
\hline $\mathbf{8 a} s$-trans $(a p)$ & 111 & 0,30 & $\mathbf{8 b} s$-trans (ap) & 113 & 0,26 \\
\hline $\mathbf{8 a} s$-trans $(s p)$ & 291 & 0,23 & $\mathbf{8 b} s$-trans $(s p)$ & 292 & 0.22 \\
\hline 9a $s$-cis (ap) & 125 & 0,51 & $\mathbf{9 b} s$-cis $(a p)$ & 126 & 0,56 \\
\hline 9a $s$-cis $(s p)$ & 302 & 0,23 & 9b $s-c i s(s p)$ & 305 & 0,25 \\
\hline 9a $s$-trans (ap) & 114 & 0,18 & $\mathbf{9 b} s$-trans $($ ap $)$ & 114 & 0,13 \\
\hline 9a $s$-trans $(s p)$ & 285 & 0,09 & 9b $s$-trans $(s p)$ & 284 & 0,06 \\
\hline
\end{tabular}


torcionais $\mathrm{C} 5-\mathrm{C} 4-\mathrm{C} 1$ '-C6' obtidos na nova otimização de geometria pelo cálculo ab initio não apresentaram diferenças significativas àquelas encontradas pela análise conformacional por AM1. Na etapa seguinte as geometrias foram completamente otimizadas, agora livres de qualquer restrição de ângulo torcional pelo método $\mathrm{HF} / 6-31 \mathrm{G}^{*}$.

A tabela 1 exibe o ângulo de diedro resultante e a fração molar para todos os compostos. As frações molares das quatro geometrias de equilíbrio dos compostos $4 \mathbf{a}-9 \mathbf{a}$ e $\mathbf{4 b - 9} \mathbf{b}$ foram determinadas usando a lei de distribuição de Bolztmann. A análise da fração molar $\left(\mathrm{x}_{\mathrm{i}}\right)$ indica uma maior proporção da estrutura $s$-cis a temperatura ambiente, com exceção do composto 8 . Os rotâmeros ap apresentam maior $x_{i}$ para a maioria dos compostos, menos para o $o_{-}$ substituído $(\mathbf{6 a}, \mathbf{b})$. Foram poucas as exceções em que a fração molar ficou abaixo de 0,1 , indicando a importância de todas as quatro geometrias no equilíbrio.

\section{Conclusões}

A análise conformacional dos compostos 4a9a e 4b-9b mostrou quatro geometrias de equilíbrio, $s$-cis/ap, s-cis/sp, s-trans/ap e $s$-trans/sp. A geometria ap apresenta ângulo torcional C4-C5-C1'-C6' próximo a $100^{\circ}$. A geometria $s p$, por sua vez, exibe diedro próximo a $280^{\circ}$, exceto para os compostos $6 \mathbf{a} \mathrm{e}$ 6b cuja inclinação do grupo arila é de $340^{\circ}$. O hamiltoniano AM1 forneceu geometrias semelhantes às obtidas pelo cálculo ab initio. A barreira envolvida na rotação da fenila é baixa. O valor encontrado no cálculo semi-emírico corresponde a, aproximadamente, $80 \%$ daquele obtido no método ab initio.

\begin{abstract}
Dihydropyrimidinones, such as monastrol and analogues, are heterocycles with known antineoplastic activity. Conformational analysis represents an important preliminary step in structure-activity correlation studies. Herein we describe the conformational analysis of monastrol and analogues by AM1 semiempirical and ab initio HF/6-31G* calculations. Four equilibrium geometries were found (s-cis/ap, s-cis/sp, strans/ap and $s$-trans $/ \mathrm{sp}$ ), and the important internal rotations were those of the $\alpha \beta$-unsaturated carbonyl system and of the aryl group connected to the heterocycle.
\end{abstract}

Keywords: conformational analysis, dihydropyrimidinones, Biginelli compound, AM1, HF/6-31G*

\section{Referências}

[1] C. O. Kappe, Eur. J. Med. Chem. 35(12) (2000) 1043.

[2] B. Desai, D. Dallinger, C. O. Kappe, Tetrahedron 62 (2006) 4651.

[3] C. O. Kappe, O. V. Shishkin, G. Uray, P. Verdino Tetrahedron 56 (2000) 1859.

[4] D. Russowsky, R. F. S. Canto, S. A. A. Sanches, M. G. M. D'Oca, A.Fátima, R. A. Pilli, L. K. Kohn, M. A. Antônio, J. E. Carvalho, Bioorg. Chem. 34 (2006) 173.

[5] E. L. Eliel, S.H. Wilen, Stereochemistry of Organic
Compounds, Wiley, New York, 1994.

[6] C.R. Rodrigues, Cadernos Temáticos Quím. Nova Esc. $3(2001) 43$.

[7] C. O. Kappe, W. M. F. Fabian, M. A. Semones, Tetrahedron 53 (1997) 2803.

[8] M.J.S. Dewar, E.G. Zoebisch, E.F. Healy, J.J.P. Stewart, J. Am. Chem. Soc. 107(13)(1985) 3902.

[9] Spartan' 02 (1.0.1), Wavefunction: Irvine, 2002.

[10] G. D. Smith, R. L. Jaffe, J. Phys. Chem. 100 (48) (1996) 18718.

M. J. Hafezi, F. Sharif, J. Mol. Struct.: THEOCHEM 814(1-3)(2007) 43. 